\title{
Phenomenological Analysis of High-Energy Heavy-Ion Collisions
}

\author{
Chiho Nonaka* \\ Kobayashi Maskawa Institute, Nagoya University, Nagoya 464-8602, Japan \\ Department of Physics, Nagoya University, Nagoya 464-8602, Japan \\ Department of Physics, Duke University, NC27705, USA \\ E-mail: nonakadhken.phys.nagova-u.ac.7p
}

\section{Kazuhisa Okamoto}

Department of Physics, Nagoya University, Nagoya 464-8602, Japan

E-mail: okamotoehken.phys.nagoya-u.ac.jp

\begin{abstract}
We develop a state-of-the-art numerical algorithm for solving the relativistic viscous hydrodynamic equation, using a Riemann solver based on two-shock approximation. We show validity of our hydrodynamics code from comparison with analytical solutions of test problems. Furthermore we perform numerical hydrodynamic calculation with a realistic fluctuating initial condition of high-energy heavy-ion collisions at LHC produced by TRENTO. We show time evolution of temperature distributions on $x-y$ and $x-\eta$ planes with lattice $\mathrm{QCD}$ equation of state (EoS) and discuss the EoS dependence of it .
\end{abstract}

The 3rd International Symposium on "Quest for the Origin of Particles and the Universe" 5-7 January 2017

Nagoya University, Japan

* Speaker. 


\section{Phenomenological Analyses of High-Energy Heavy-Ion Collisions}

At present to gain insight into Quark-Gluon Plasma (QGP), high-energy heavy-ion collision experiments are performed at Relativistic Heavy Ion Collider (RHIC) and LHC (Large Hadron Collider). The QGP is a new state of matter of quarks and gluons at extremely high temperature and/or density. One of the most important outcomes in QGP physics is that the strongly interacting QGP (sQGP) was created at RHIC [U]. The discovery of the sQGP brought us a chance to look into a new aspect of the QGP, because the QGP had been expected to be a gas, weakly interacting QGP, before the RHIC operation. At that time, the sQGP production at RHIC is confirmed from both experimental and theoretical studies: success of relativistic hydrodynamics for explaining strong elliptic flow, quark number scaling in elliptic flow which is understood by a recombination model, jet quenching mechanism for the suppression of nuclear modification factor and color class condensate for initial state [四]. In particular, since then a relativistic hydrodynamic model has been one of promising dynamical models for description of space-time evolution of hot and dense matter created after high-energy heavy-ion collisions.

In this decade, development of hydrodynamic model itself is remarkable [[]]. First a $(2+1)$ dimensional relativistic viscous hydrodynamic model appeared. They focused on physics around the mid rapidity and solved the relativistic viscous hydrodynamic equation only on transverse plane, using Israel-Stewart theory. Inclusion of viscosity into the relativistic hydrodynamic equation is not easy task. If viscosities are included in the first order of gradient, then acausality happens. Other than the Israel-Stewart theory, several possible relativistic viscous hydrodynamic equations in the second order of gradient have been proposed. Here the problem is that we do not reach a conclusion which relativistic viscous hydrodynamic equation is suitable for description of high-energy heavy ion collisions.

Now higher harmonics as well as direct and elliptic flows attracts a lot of interest, because it gives as a clue to understand the detailed QGP bulk property such as shear and bulk viscosities from comparison between experimental results and theoretical calculations. The origin of higher harmonics is considered as existence of fluctuations in initial state and development of it depends on hydrodynamic expansion. Therefore the numerical calculation with high precision is indispensable to evaluate correctly the higher harmonics. However the importance of numerical algorithm for the solving relativistic viscous hydrodynamic equation has not been paid attention.

Here we construct a state-of-the-art numerical algorithm with the Riemann solver based on the two shock approximation [ [ 6 , 目]. We check the correctness of our relativistic hydrodynamics code from comparison with several analytical solutions. We also confirm the energy and momentum conservation in one-dimensional expansion of high-energy heavy ion collisions. Using our hydrodynamic model, we investigate possible existence of Kelvin-Helmholtz instability in high-energy heavy ion collisions $[\square]$. Finally we perform numerical hydrodynamic calculation with realistic initial condition to $\mathrm{Pb}+\mathrm{Pb}$ collisions at LHC. 


\section{Relativistic Hydrodynamic Model}

\subsection{A New Relativistic Hydrodynamics Code}

A relativistic hydrodynamic code is developed for solving the conservation equations

$$
\begin{aligned}
N_{; \mu}^{\mu} & =0, \\
T_{; \mu}^{\mu \nu} & =0,
\end{aligned}
$$

where $N^{\mu}$ is the net charge current and $T^{\mu v}$ is the energy-momentum tensor. Choosing the Landau frame, we decompose the net charge current and the energy-momentum tensor of the viscous fluid as

$$
\begin{aligned}
N^{\mu} & =n u^{\mu}+n^{\mu}, \\
T^{\mu v} & =e u^{\mu} u^{v}-(p+\Pi)\left(g^{\mu v}-u^{\mu} u^{v}\right)+\pi^{\mu v},
\end{aligned}
$$

where $n^{\mu}$ is the charge diffusion current, $\Pi$ is the bulk pressure, and $\pi^{\mu v}$ is the shear tensor.

We develop the relativistic hydrodynamics code in the Milne coordinates which are suitable for description of strong longitudinal expansion at RHIC and LHC. In our algorithm, we split the conservation equations Eq. (․․) and Eq. (2.2) into two parts, an ideal part and a viscous part using the Strang splitting method $[8]$. For the ideal part we use a Riemann solver with the two shock approximation [B]. In the viscous part, if the relaxation times are much shorter than the fluid time scale, we utilize the Piecewise Exact Solution (PES) method [䧃] to save the computational time.

We check the correctness of our code in several test problems [ [0, []. Here we show highlights of them: the conservation property of our code and the Israle-Stewart theroy in the Gubser flow regime. First we check the conservation property of our hydrodynamics codes, using a fluctuating initial condition in the $\eta$ direction. In this calculation we switch off viscosity effect to know how large the numerical viscosity itself exists in our algorithm.

$$
\begin{aligned}
e\left(\tau_{0}, \eta\right) & =e^{\text {flat }}\left(\tau_{0}, \eta\right) \times\left(1+\sum_{n=0}^{10} \delta e_{n} \cos \left(n \frac{2 \pi\left(\eta-\eta_{n}^{e}\right)}{L}\right)\right), \\
w^{\eta}\left(\tau_{0}, \eta\right) & =\sum_{n=0}^{10} \delta w_{n}^{\eta} \cos \left(n \frac{2 \pi\left(\eta-\eta_{n}^{v}\right)}{L}\right),
\end{aligned}
$$

values of $\eta_{n}^{e}$ and $\eta_{n}^{v}$ are chosen between $\eta=-Y_{b}$ and $Y_{b}$ at random and $e^{\text {flat }}\left(\tau_{0}, \eta\right)$ is given by

$$
e^{\text {flat }}\left(\tau_{0}, \eta\right)=e_{0} \exp \left[-\frac{\left(\left|\eta_{s}\right|-\eta_{\text {flat }} / 2\right)^{2}}{\sigma_{\eta}^{2}} \theta\left(|\eta|-\eta_{\text {flat }} / 2\right)\right] \times \theta\left(Y_{b}-|\eta|\right) .
$$

Here $Y_{b}=5.3$ is the beam rapidity, $\sigma_{\eta}=2.1$ and $\eta_{\text {flat }}=2.6$ show the size of the flat structure of the initial energy density distribution in the rapidity and $e_{0}=30 \mathrm{GeV} / \mathrm{fm}^{3}$ is the maximum value of the energy density. We choose a typical parameter set which is tuned for the RHIC collision energy [ 6 ]. We carry out numerical calculations with the grid size $\Delta \eta=0.2$ which is often chosen in calculation of high-energy heavy-ion collisions. We set the time-step size to $\Delta \tau=0.1 \tau_{0} \Delta \eta$. In Fig.W the energy density and velocity distributions at $\tau=10 \mathrm{fm}$ with and without the source terms are shown. At 
the mid-rapidity the energy density and the flow distributions of numerical calculations with and without the source terms are consistent with each other. In the region of $|\eta|>4$, however, the differences between them are observed in the small structure of both distributions. The growth of the velocity to the vacuum $|\eta| \sim 8$ gives a difficulty of numerical calculation and becomes the reasons for the differences.

The deviation from the energy and momentum conservation is listed in Tab.W. For both cases, we find that they are around ten times as large as those with the smoothed initial condition. Nevertheless, the code based on the conservative form keeps conservation property with high accuracy. On the other hand, in the code with the source terms a few \% deviation from the energy and momentum conservation appears, which is still acceptable.
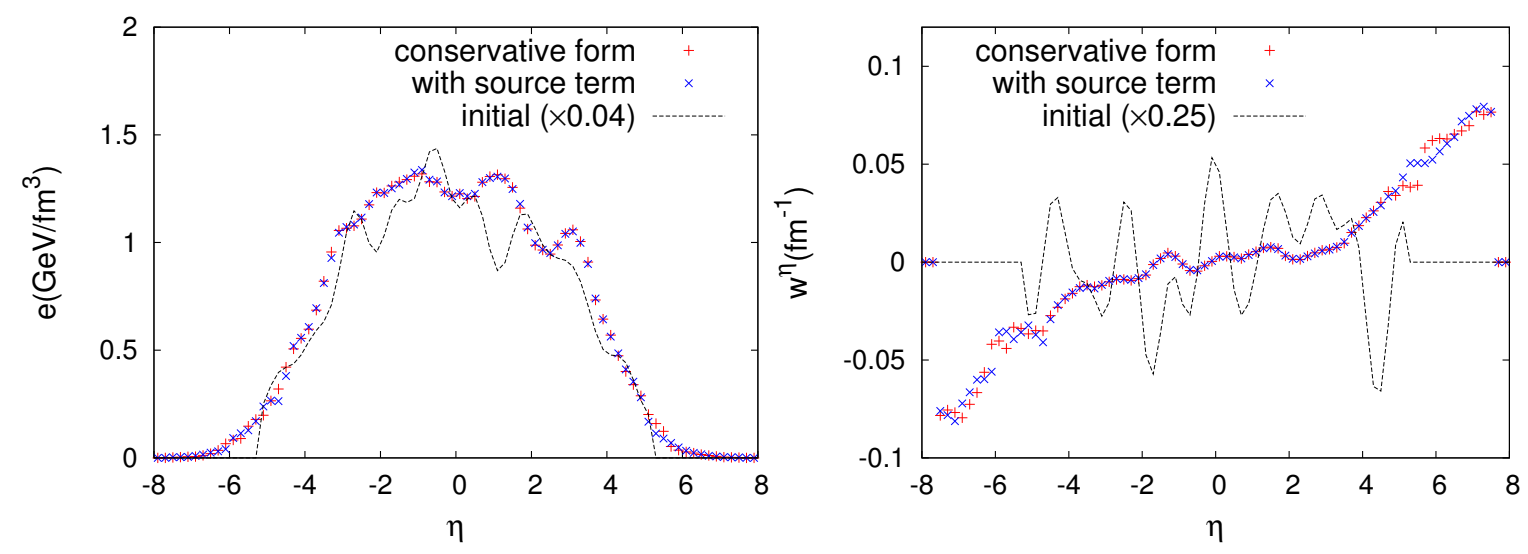

Figure 1: The numerical results for longitudinal expansion with the fluctuating initial condition at $\tau=10$ fm. Left: The energy distributions from the codes with the conservative form and with the source terms. The dotted line stands for the initial energy distribution. Right: The velocity distribution from the codes with the conservative form and with the source terms. The dotted line stands for the initial velocity distribution.

\begin{tabular}{c|cc}
\hline & $\varepsilon_{E}$ & $\varepsilon_{M}$ \\
\hline conservative & $1.38 \mathrm{E}-09$ & $8.59 \mathrm{E}-09$ \\
with souce & $1.27 \mathrm{E}-02$ & $5.61 \mathrm{E}-02$ \\
\hline
\end{tabular}

Table 1: The violation of the total energy and momentum conservation with fluctuating initial conditions. .

Next we show comparison between our numerical calculation and analytical solution of the Israel-Stewart theory in the Gubser flow regime. A semi-analytic solution of the Israel-Stewart theory in the Gubser flow regime is discussed in Ref.[Q], based on the symmetry arguments developed by Gubser [山, $\mathbb{1 0}$ ]. The numerical calculation is performed on the space-grid size $(\Delta x, \Delta y, \Delta \eta)=$ $(0.05 \mathrm{fm}, 0.05 \mathrm{fm}, 0.1)$ with the time-step size $\Delta \tau=0.1 \Delta x$. The shear viscosity and the relaxation time are set to $\eta / s=0.2$ and $\tau_{\eta}=5 \eta /(T s)$, respectively.

Figure $\square$ shows the time evolution of the temperature and $x$ component of fluid velocity as a function of $x$ from the numerical results and the semi-analytic solutions. In the case of the inviscid fluid, the numerical calculation follows the analytical solution until $\tau=7 \mathrm{fm}$ [目], whereas in the 
viscous fluid the difference between numerical results and the semi-analytic solution appears after $\tau=4 \mathrm{fm}$. We show the time evolution of shear tensors in Fig. B. The shear tensors $\pi^{x x}, \pi^{y y}$ and $\pi^{\eta \eta}$ in our numerical calculations show good agreement with the semi-analytic solutions. However, in $\pi^{x y}$ the deviation from the semi-analytic solution starts to appear at $\tau=2 \mathrm{fm}$ and grows at later time. Furthermore we find that the second-order terms in $\pi^{\mu v}$ give us a clue of choice of numerical scheme for evaluation of the convection term in the Israle-Stewart theroy [प].
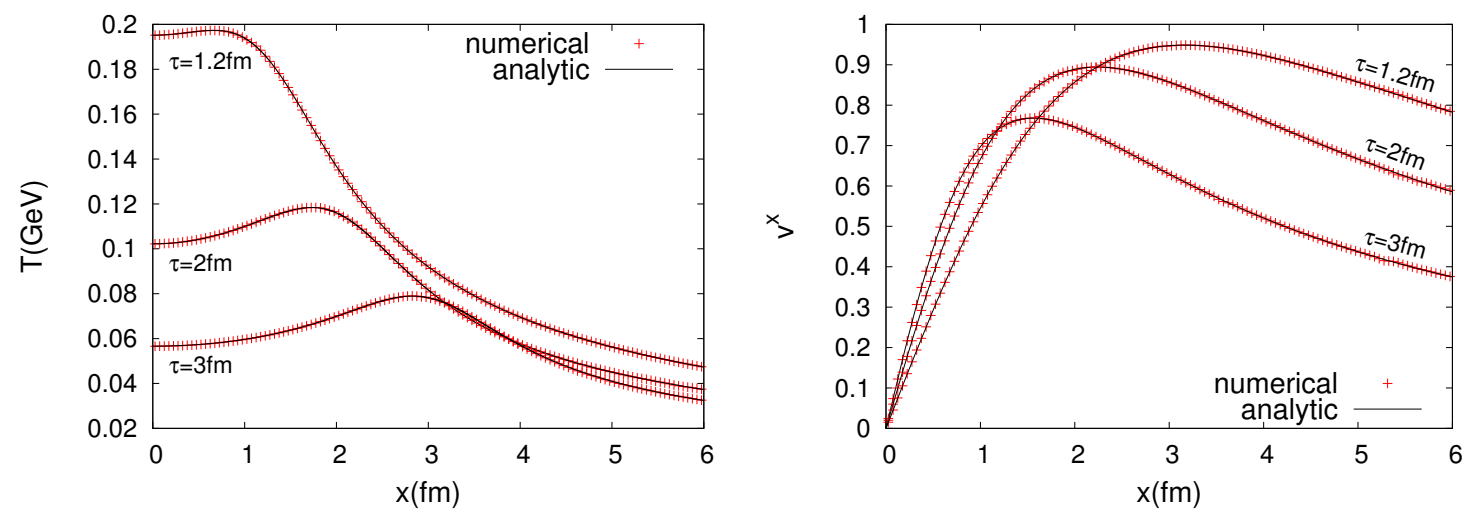

Figure 2: The time evolution of temperature $T$ (left panel) and the $x$ component of fluid velocity $v^{x}$ (right panel) distribution as a function of $x$. The solid lines stand for the semi-analytic solutions and the pluses stand for numerical results.

\subsection{Toward Analyses of High-Energy Heavy-Ion Collisions}

We apply our relativistic viscous hydrodynamics code to analyses of high-energy heavy-ion collisions. First we need to prepare an appropriate initial condition which can reproduce the experimental data. We employ a parametric initial-condition model "TRENTO (the Reduced Thickness Event-by-event Nuclear Topology model)" based on eikonal entropy deposition via "reducedthickness" function [12]. In the model, entropy density is proportion to

$$
s \propto\left(\frac{T_{A}^{p}+T_{B}^{p}}{2}\right)^{1 / p},
$$

where $T_{A}^{p}$ and $T_{B}^{p}$ are the thickness functions,

$$
T_{A, B}^{p}(x, y)=w_{A, B} \int d z \rho_{A, B}(x, y, z)
$$

with independent random weights $w_{A, B}$ sampled from a gamma distribution [12]. The interesting feature of the initial condition is that it mimics current proposed initial conditions changing the parameter $p$. For example, if $p=1$, the entropy is proportion to $s \propto T_{A}+T_{B}$ which corresponds to the wounded nuclear model. On the other hand, if $p=0$, the entropy follows $s \propto \sqrt{T_{A} T_{B}}$ which is similar to entropy density of IP-Glasma and EKRT model.

Left panel of Figs. $\$$ and $\square$ show the initial temperature distributions for $\sqrt{s_{N N}}=2.76 \mathrm{GeV}$ $\mathrm{Pb}+\mathrm{Pb}$ central collisions $(b \sim 1 \mathrm{fm})$ on the $x-y$ plane and $x-\eta$ plane produced by TRENTO. We transform the entropy density distributions from the TRENTO to the temperature distributions 

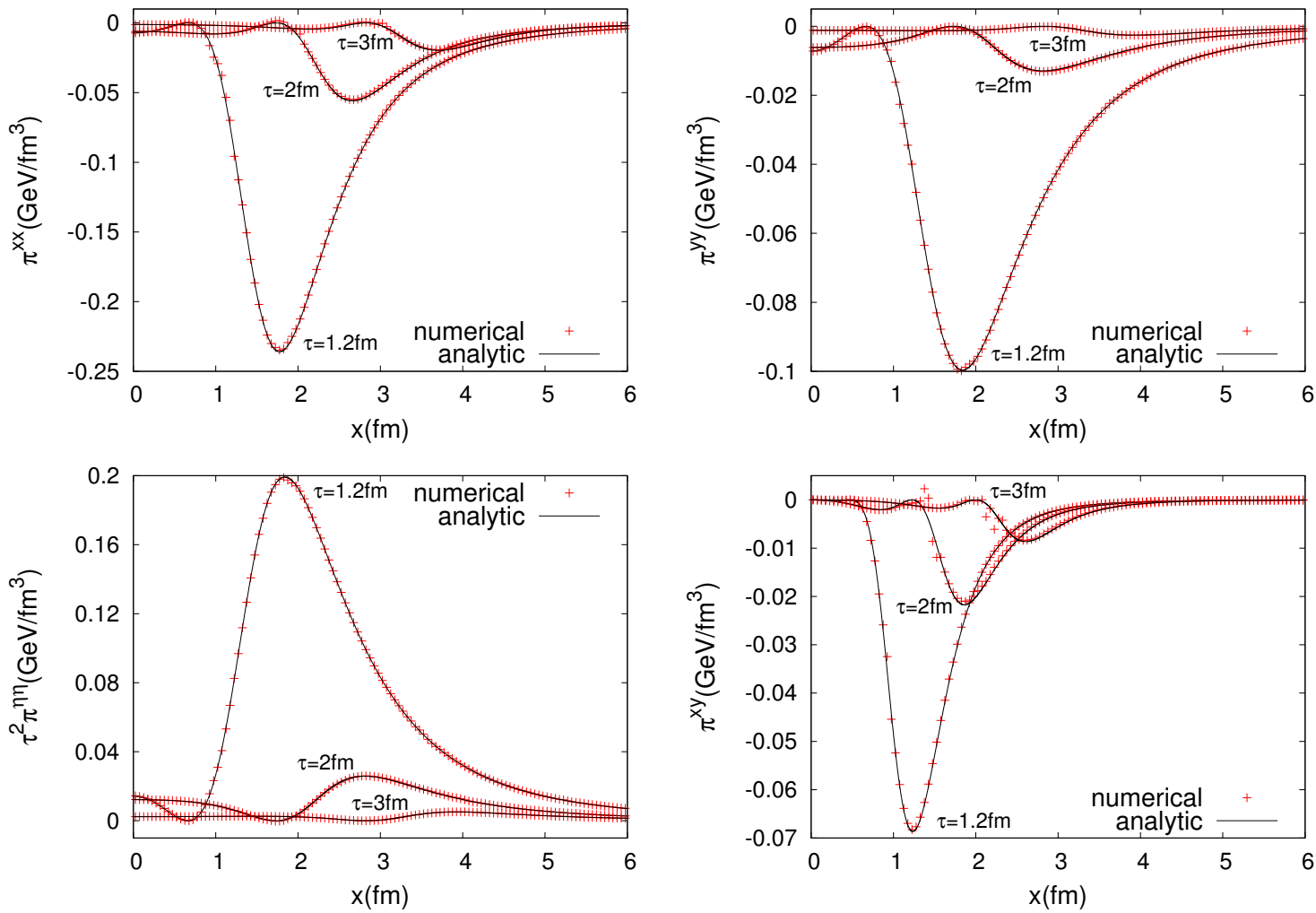

Figure 3: The time evolution of shear tensors $\pi^{x x}$ (top left), $\pi^{y y}$ (top right), $\tau^{2} \pi^{\eta \eta}$ (bottom left) and $\pi^{x y}$ (bottom right) from the Gubser fliow as a function of $x$. The solid lines stand for the semi-analytic solutions and the pluses stand for numerical results.

using the equation of state (EoS) of the Lattice QCD [14]]. In the left panel of Fig. Ty we can see the granularity structure whose size is around $2 \mathrm{fm}$. The highest temperature of them is $\sim 600 \mathrm{MeV}$. The absolute value of the entropy density is one of parameters of TRENTO. We need to fix the parameter from comparison with experimental data like transverse momentum distributions and rapidity distributions. On the other hand, the initial temperature on the $x-\eta$ plane has several string-like structures, which suggests a consequence of the existence of strong expansion in the longitudinal direction.

The right figures of Figs. $[$ and 5 show the temperature distributions on the $x-y$ and the $x-\eta$ planes at $\tau=10 \mathrm{fm}$. In the calculation we use the lattice QCD EoS and set the shear viscosity to $\eta / s=0.2$. In the right panel of Fig.⿴囗十, the initial granularity structure is smeared but there is still a rough structure which seems to contain superposition of harmonics, $v_{1}, v_{2}, v_{3}$ and more. Whereas in the temperature distribution on the $x-\eta$ plane, string-like structures are almost gone and smoothed distributions remain.

In Fig. We make a comparison between the temperature distribution with ideal gas EoS and that with lattice QCD EoS at $\tau=5$ and $8 \mathrm{fm}$. In the comparison we use the same initial entropy density distribution from TRENTO. We can see the difference of EoS affects not only initial 

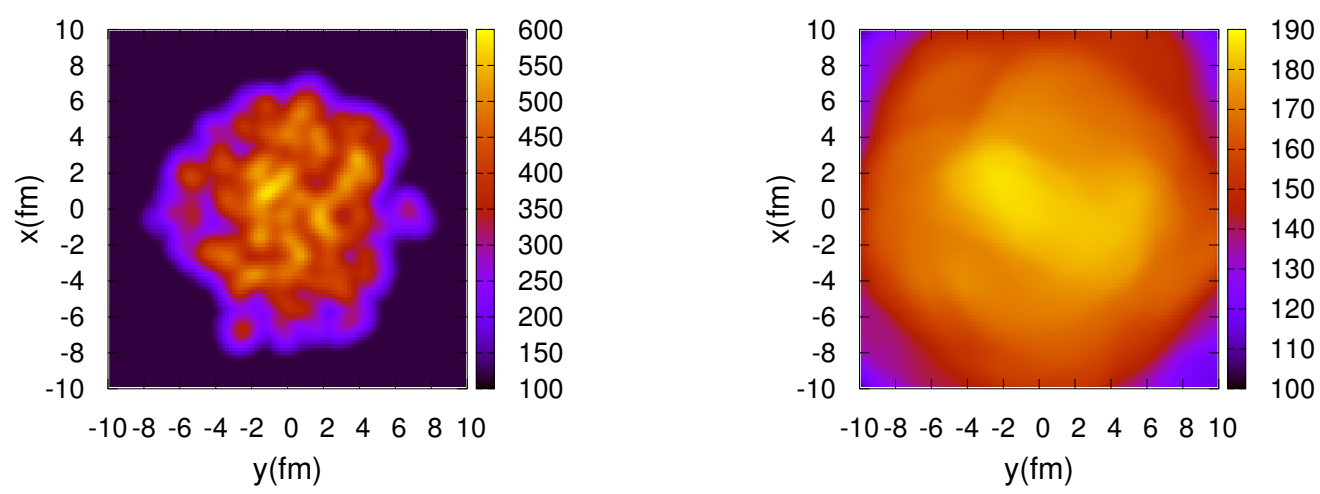

Figure 4: The temperature distributions on the $x-y$ plane at $\tau=1 \mathrm{fm}$ (left) and at $\tau=10 \mathrm{fm}$ (right).
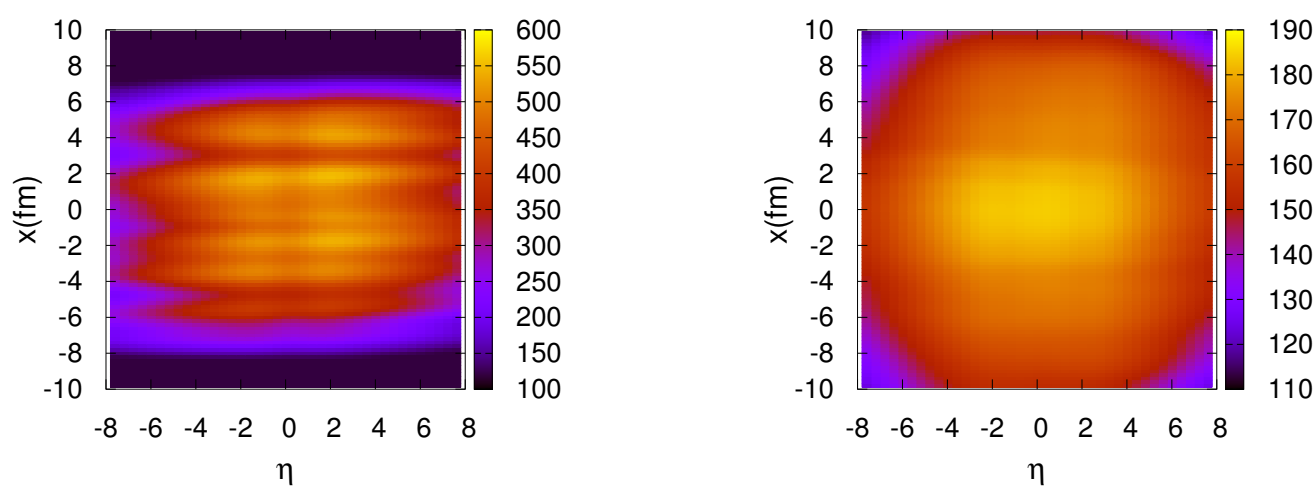

Figure 5: The temperature distributions on the $x-\eta$ plane at $\tau=1 \mathrm{fm}$ (left) and at $\tau=10 \mathrm{fm}$ (right).

temperature distribution but also hydrodynamic expansion. Generally the value of temperature distribution with lattice QCD EoS is higher than that with ideal gas EoS, which suggests that the lifetime of QGP fluid with lattice QCD EoS is longer than that with ideal gas EoS.

Figure $\square$ shows the freezeout hypersurface as a function of $x \mathrm{fm}$ (left) and $\eta$ (right). Here we assume that the freezeout process occurs if the temperature of the fluid cell is below $T_{f}=155$ $\mathrm{MeV}$. Using the information of temperature and fluid velocity on the freezeout hypersurface, we calculate the particle distribution which is an initial condition for hadron based event generator such as UrQMD. We leave further calculation to a future project. Here we confirm that our numerical algorithm is stable and solid even with realistic fluctuating initial condition for high-energy heavy ion collisions. Now we are ready for analyses of experimental data at RHIC and LHC. 


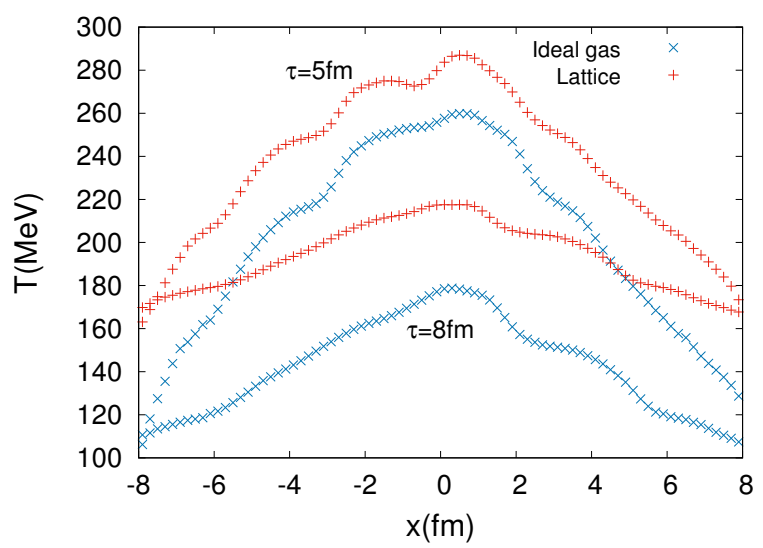

Figure 6: The temperature distributions as a function of $x(\mathrm{fm})(y=\eta=0)$ at $\tau=5$ and $8 \mathrm{fm}$ in the case of lattice QCD EoS (the red points) and ideal gas EoS (the blue points).
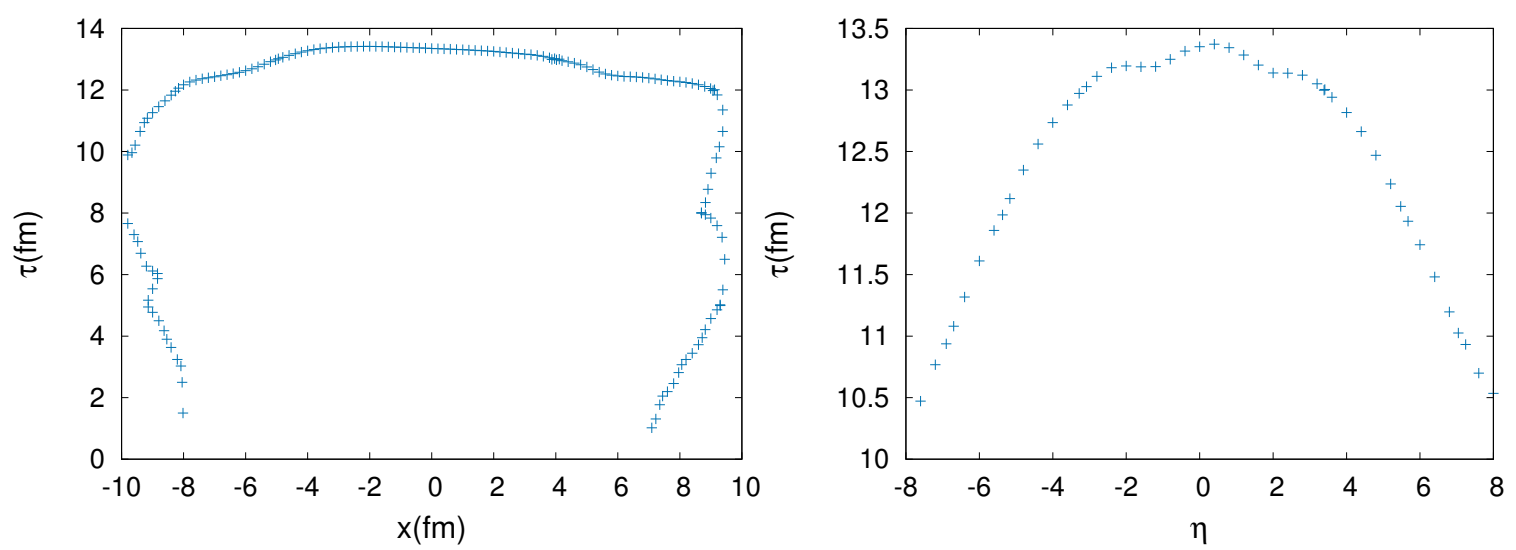

Figure 7: The freezeout hypersurface as a function of $x(\mathrm{fm})(y=\eta=0)$ (left) and $\eta x=y=0$ (right).

\section{Summary}

We have developed the state-of-the-art numerical algorithm for solving the relativistic viscous hydrodynamic equation, using the Riemann solver based on two-shock approximation. Our hydrodynamics code showed the good property in the total energy and momentum conservation even with fluctuating initial condition, which is very important feature for analyses of higher harmonics at RHIC and LHC. Furthermore we have shown comparison between our numerical calculation and analytical solution of the Israel-Stewart theory in the Gubser flow regime. Not only time evolution of temperature and velocity but also that of the shear tensors in our numerical calculations show good agreement with the semi-analytic solutions. Finally we performed numerical hydrodynamic calculation with the realistic fluctuating initial condition of high-energy heavy-ion collisions produced by TRENTO. We found that our numerical hydrodynamics code with the initial conditions 
is stable enough to follow the entire time evolution of system until the temperature of the all fluid cells becomes below the freezeout temperature. We showed the time evolution of temperature distributions on $x-y$ and $x-\eta$ planes with lattice QCD EoS. We investigated the EoS dependence of time evolution of temperature distributions. In the case of lattice QCD EoS, the life time of the QGP fluid is longer than that with the ideal gas EoS: the values of initial temperature distributions are higher than those with the ideal gas EoS and temperature decreasing rate is slower. We presented the freezeout hypersurfaces which are used for calculation of particle yields. Now further calculation is proceeding for comparison with experimental data at RHIC and LHC.

\section{Acknowledgments}

The work of C.N. is supported by the JSPS Grant-in-Aid for Scientific Research (S) No. 26220707 and US Department of Energy grant DE-FG02-05ER41367.

\section{References}

[1] Quark Gluon Plasma. New discoveries at RHIC: Case for the Strongly Interacting Quark- Gluon Plasma., Nucl. Phys. A750, 1 (2005).

[2] C. Nonaka and M. Asakawa, PTEP 2012, 01 A208 (2012) doi:10.1093/ptep/pts014 [arXiv:1204.4795 [nucl-th]].

[3] A. Mignone, T. Plewa, G. Bodo, ApJS 160, 199 (2005). doi:10.1086/430905 arXiv:astro-ph/0505200.

[4] M. Takamoto and S. Inutsuka, J. Comput. Phys. 11, 38 (2011). doi:10.1016/j.jcp.2011.05.030 arXiv:1106.1732 [astro-ph].

[5] Y. Akamatsu, S. i. Inutsuka, C. Nonaka and M. Takamoto, J. Comput. Phys. 256 (2014) 34 doi:10.1016/j.jcp.2013.08.047 [arXiv:1302.1665 [nucl-th]].

[6] K. Okamoto, Y. Akamatsu and C. Nonaka, Eur. Phys. J. C 76 (2016) no.10, 579 doi:10.1140/epjc/s10052-016-4433-x [arXiv:1607.03630 [nucl-th]].

[7] K. Okamoto and C. Nonaka, arXiv:1703.01473 [nucl-th].

[8] G. Strang, SIAM J. Numer. Anal. 5, 506 (1968) doi:10.1137/0705041.

[9] H. Marrochio, J. Noronha, G. S. Denicol, M. Luzum, S. Jeon, and C. Gale, Phys. Rev. C 91, 014903 (2015). doi:10.1103/PhysRevC.91.014903[ arXiv:1307.6130 [nucl-th]].

[10] S. S. Gubser, Phys. Rev. D 82, 085027 (2010). doi:10.1103/PhysRevD.82.085027 [arXiv:1006.0006 [hep-th]].

[11] S. S. Gubser and A. Yarom, Nucl. Phys. B846, 469 (2011). doi:10.1016/j.nuclphysb.2011.01.012 [arXiv:1012.1314 [hep-th]].

[12] J. S. Moreland, J. E. Bernhard and S. A. Bass, Phys. Rev. C 92 (2015) no.1, 011901 doi:10.1103/PhysRevC.92.011901 [arXiv:1412.4708 [nucl-th]].

[13] J. E. Bernhard, J. S. Moreland, S. A. Bass, J. Liu and U. Heinz, Phys. Rev. C 94 (2016) no.2, 024907 doi:10.1103/PhysRevC.94.024907 [arXiv:1605.03954 [nucl-th]].

[14] M. Bluhm, P. Alba, W. Alberico, A. Beraudo and C. Ratti, Nucl. Phys. A 929 (2014) 157 doi:10.1016/j.nuclphysa.2014.06.013 [arXiv:1306.6188 [hep-ph]]. 